\title{
Comparaison des relations trophiques de Ruditapes philippinarum en milieux estuarien et océanique à partir d'un modèle de croissance
}

\author{
C. Bacher et P. Goulletquer \\ Laboratoire national écosystèmes conchylicoles (L.E.C.) IFREMER, Mus de Loup, B.P. 13317390 LA TREMBLADE (France)
}

Bacher, C. et P. Goulletquer. 1989. Comparaison des relations trophiques de Ruditapes philippinarum en milieux estuarien et océanique à partir d'un modèle de croissance. Can. J. Fish. Aquat. Sci. 46: 1160-1170.

\begin{abstract}
Cette étude porte sur l'établissement des relations trophiques de Ruditapes philippinarum en zone estuarienne et océanique du bassin de Marennes-Oléron au cours de l'année 1985-1986. L'utilisation de I'analyse en composantes principales permet de décrire, à partir des axes principaux, les fluctuations des paramètres environnementaux. Les variations de poids sec sont expliquées à partir d'un modèle de croissance allométrique utilisant les axes principaux de l'analyse en composantes principales. Les croissances observées et simulées sont supérieures en site océanique. Une déplétion de nourriture apparaît en période de floraison phytoplanctonique automnale entre les deux sites. Les remises en suspension du sédiment, provoquant de fortes turbidités, limiteraient la croissance en zone estuarienne. Les problèmes de la stratégie d'échantillonnage et de l'échelle temporelle des relations trophiques sont posés à partir des résultats du modèle.
\end{abstract}

A comparison of trophic relationships for Ruditapes philippinarum in estuarine and oceanic areas: the use of a growth model. Trophic relationships in Ruditapes philippinarum were studied in estuarine and oceanic areas of Marennes-Oleron during 1985 and 1986. Principal components analysis (P.C.A.) was used to reveal the fluctuations of the descriptors from the principal axes. Changes in dry weight were predicted in each area through an allometric growth model and were related to the principal axes derived from the P.C.A. The results of the model were used to compare the growth in each area and to point out the descriptors responsible for the discrepancies, such as autumnal blooms or silt resuspension. Attention was paid to the sampling strategy related to the time scale of the phenomena accounting for trophic relationships.

Reçu le 25 mai 1988

Received May 25, 1988

Accepté le 17 février 1989

(J9757)

Accepted February 17, 1989

L

a palourde japonaise Ruditapes philippinarum, introduite dans les années 1970, fait actuellement l'objet d'une aquaculture en plein essor sur le littoral français (600 t en 1987). En Charente-Maritime, l'activité s'est particulièrement développée depuis 1980 , suite à la mise au point des techniques d'élevage dans les marais ostréicoles (Peyre et al. 1980). Toutefois, en raison d'une capacité trophique limitée des claires ostréicoles (Goulletquer et al. 1988), les densités d'élevage restent limitées à $500 \mathrm{~g} / \mathrm{m}^{2}$. Une nouvelle expansion de cet élevage nécessite de s'orienter vers les bassins conchylicoles et d'étudier les relations trophiques en fonction des sites de nature estuarienne ou océanique.

L'étude expérimentale des réponses physiologiques des suspensivores a permis de montrer que les taux de croissance sont régulés par l'interaction de facteurs environnementaux, dont la qualité et la quantité de nourriture (Bayne et Widdows 1978 ; Bayne et al. 1987). Toutefois, peu d'études ont abordé les relations trophiques in situ existant entre les paramètres du milieu et l'animal (voir synthèse Héral et al. 1987).

Généralement, les interactions synergiques des variables environnementales compliquent l'interprétation de l'importance de chaque facteur du milieu (Page et Hubbard 1987). Fréchette et Bourget (1985a) mettent en évidence les différences de croissance chez Mytilus edulis selon la position dans la colonne d'eau et le niveau intertidal dont les effets sont interprétés en termes de déplétion de la nourriture potentielle dis- ponible. Berg et Newell (1986) associent la variabilité des croissances annuelles de Crassostrea virginica à des différences de quantités moyennes de nourriture disponible. Dans le but d'affiner l'étude du couplage croissance-nourriture potentielle, il semble donc intéressant de quantifier les relations trophiques en tenant compte de la variabilité spatio-temporelle du milieu.

Récemment, l'utilisation de méthodes statistiques de type régression multiple a permis d'établir des relations trophiques à partir de descripteurs du milieu, en les classifiant par ordre d'importance (Héral et al. 1984 ; Page et Hubbard 1987; Parache et Massé 1987). L'hypothèse de la linéarité des relations était retenue dans le cadre de ces études.

La presente étude a pour but d'étendre ces relations au cas de non-linéarité par une modélisation des variations du poids sec de l'animal, à partir des observations hydrologiques et sédimentaires du milieu durant deux années. Les propriétés du modèle permettent de classifier les variables du milieu en fonction de leur effet sur la croissance de Ruditapes philippinarum en zone estuarienne et océanique. Une approche prédictive permet également de quantifier l'effet du temps d'immersion sur les performances de croissance.

\section{Lieu d'expérimentation et méthodes}

L'étude des relations trophiques de Ruditapes philippinarum a été effectuée sur deux sites du bassin de Marennes-Oléron 



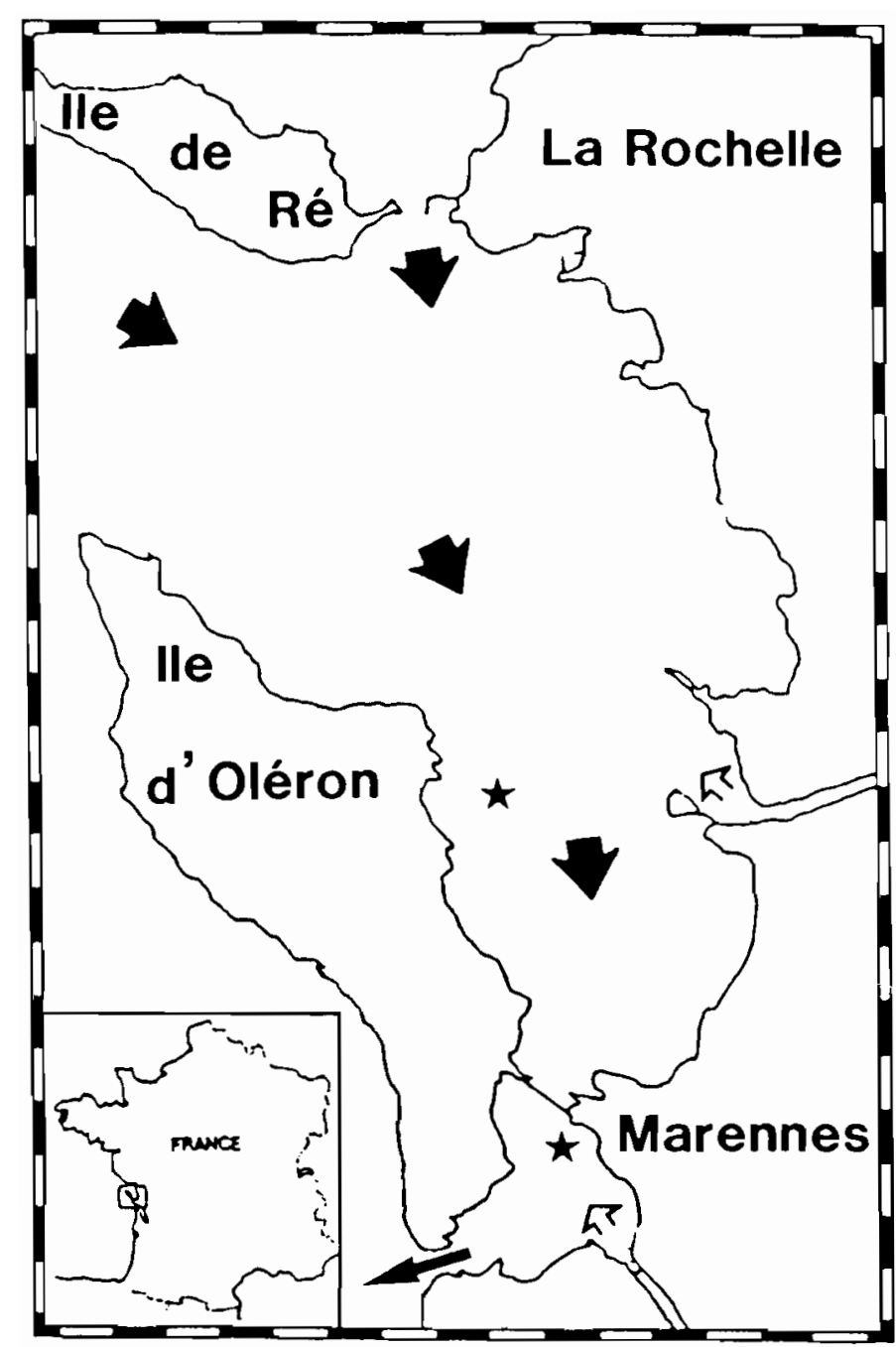

F1G. 1. Localisation des sites expérimentaux $(\star)$ et circulation globale des masses d'eaux océaniques (\$) et fluviales $(\emptyset)$ dans le bassin de Marennes-Oléron.

(fig. 1) : sous influence océanique, dans la partie nord du bassin, le site de Lilleau a pour substrat d'élevage un sédiment de type sableux ( $3 \%$ pélites) et un temps d'immersion journalier moyen de $21 \mathrm{~h}$. Le second site, au substrat de type vaseux (90\% pélites) et à temps d'immersion de $18 \mathrm{~h}$ est localisé près de l'Ile de Nole, dans la partie médiane du bassin ostréicole en zone estuarienne. Les vitesses de courant sont par ailleurs du même ordre de grandeur sur les 2 sites $\left(30 \mathrm{à} 40 \mathrm{~cm} \cdot \mathrm{s}^{-1}\right)$. La circulation générale des eaux d'origine océanique et estuarienne (fig. 1) dans le bassin suit une résultante nord-sud, avec un temps de résidence de l'ordre de 5 à $9 \mathrm{~d}$ en fonction des oscillations tidales. Afin d'étudier les relations trophiques de l'espèce, les suivis portent sur les fluctuations de la croissance de l'animal, des paramètres hydrologiques et de l'interface eau-sédiment. Les paramètres organiques sédimentaires peuvent en effet représenter une nourriture potentielle pour les palourdes selon Bodoy et Plante-Cuny (1984) et Plante-Cuny et Bodoy (1987).

\section{a) Stratégie d'échantillonnage}

Pour mettre en relation la production du mollusque et les variables hydrologiques et sédimentaires du milieu, il est nécessaire d'obtenir la meilleure représentation possible du milieu. $\mathrm{Si}$, pour l'animal, des mesures mensuelles ou bimensuelles des productions tissulaires permettent d'intégrer les fluctuations du milieu, le problème reste de définir un intervalle de temps et une stratégie d'échantillonnage appropriée au couplage benthos-pelagos (Héral et al. 1987).

La variabilité observée sur les paramètres hydrologiques en zone estuarienne est de quatre ordres : la stratification entre la surface et le fond de la colonne d'eau ; le cycle journalier dû à la marée ; le cycle mensuel de mortes eaux et de vives eaux ; la tendance saisonnière.

La stratégie adoptée vise à estimer les moyennes mensuelles non biaisées des variables considérées : l'échantillonnage hydrologique s'effectue en surface et au fond de la colonne d'eau, à proximité des zones d'élevage avec un intervalle de temps de $1 \mathrm{~h} 30$ min pendant un demi-cycle de marée, en période de vives eaux et de mortes eaux. Une précision de l'ordre de $5 \%$ de la moyenne journalière est ainsi obtenue avec cet intervalle de temps (Héral et al. 1989).

Les paramètres sédimentaires sont estimés à partir de prélèvements effectués sur chaque site d'élevage en période de vives eaux et de mortes eaux. Trois prélèvements sont réalisés sur la surface considérée afin de tenir compte de la variabilité spatiale.

\section{b) Paramètres du milieu}

En hydrologie, neuf paramètres sont retenus afin de caractériser la masse d'eau : température (Temp), saturation en oxygène $\left(\mathrm{HO}_{2} \mathrm{P}\right)$, protéines (HPRO), lipides (HLIP) et carbohydrates particulaires (HCAR), chlorophylles $a$ (HCHL) et phéopigments (HPHE), seston minéral (HPIM) et organique (HPOM). Les prélèvements et les analyses sont effectués selon les protocoles décrits par Héral et al. (1984).

L'étude du sédiment est effectuée par raclage du premier centimètre. Six paramètres sont retenus pour caractériser la nourriture potentielle : quantité de matière organique (SPOM), chlorophylle $a$ (SCHL), phéopigments (SPHE), protéines (SPRO), lipids (SLIP) et carbohydrates particulaires (SCAR). Les protocoles sont décrits en détail par Héral et al. (1984).

\section{c) Population d'élevage}

Les animaux de taille comparable $(\overline{\mathrm{L}}=27,5 \mathrm{~mm}, \overline{\mathrm{Pds}}=$ $4,73 \mathrm{~g}$ ) sont semés sur les deux sites en avril 1985 à une densité de 250 ind.$/ \mathrm{m}^{2}$ sur une surface de $1000 \mathrm{~m}^{2}$. Le suivi de croissance des palourdes, réalisé jusqu'en juillet 1986, est estimé par des échantillons bimensuels ou mensuels de 20 individus prélevés au hasard dont le poids sec de chair est obtenu par lyophilisation pendant $24 \mathrm{~h}$.

L'émission des gamètes, n'étant pas explicable dans le cadre de l'étude des relations trophiques, nous amène à considérer deux périodes de croissance : la classe 1 d'avril à octobre 1985 et la classe 2 de novembre 1985 à juillet 1986 .

\section{Traitement statistique}

\section{Analyse factorielle}

Les interactions existant entre les variables hydrosédimentaires descriptives du milieu ne permettent pas de préciser l'influence de chaque variable sur la croissance (tableau 1). L'explication de celle-ci nécessite par conséquent la recherche de variables explicatives synthétiques et indépendantes. L'utilisation de l'analyse factorielle permet de normaliser et d'identifier des groupes de variables corrélées 
TABLEAU 1. Matrice de corrélation entre les 15 variables. Hydrologie : HPRO = protéines, HLIP = lipides, HCAR = carbohydrates, HPIM $=$ seston minéral, $\mathrm{HPOM}=$ seston organique, $\mathrm{HCHL}=$ chlorophylle $a, \mathrm{HPHE}=$ phéopigments, $\mathrm{TEMP}=$ température, $\mathrm{HO}_{2} \mathrm{P}=$ pourcentage de saturation en oxygène. Sédiment: $\mathrm{SPOM}=$ matière organique, SCHL $=$ chlorophylle $a, \mathrm{SPHE}=$ phéopigments, SPRO $=$ protéines, $\mathrm{SLIP}$ = lipides, SCAR = carbohydrates.

\begin{tabular}{|c|c|c|c|c|c|c|c|c|c|c|c|c|c|c|c|}
\hline & \multicolumn{9}{|c|}{ Hydrologie } & \multicolumn{6}{|c|}{ Sédiment } \\
\hline & H Pro & H Lip & H Car & H Pim & H Pom & H Chl & H Phe & Temp & $\mathrm{HO}_{2} \mathrm{P}$ & S Pom & S Chl & S Phe & S Pro & S Lip & S Carb \\
\hline H Pro & 1,000 & & & & & & & & & & & & & & \\
\hline H Lip & 0,211 & 1,000 & & & & & & & & & & & & & \\
\hline H Car & 0,852 & 0,110 & 1,000 & & & & & & & & & & & & \\
\hline H Pim & 0,804 & 0,031 & 0,915 & 1,000 & & & & & & & & & & & \\
\hline H Pom & 0,654 & 0,038 & 0,824 & 0,890 & 1,000 & & & & & & & & & & \\
\hline H Chl & 0,009 & 0,061 & $-0,026$ & $-0,279$ & $-0,256$ & 1,000 & & & & & & & & & \\
\hline H Phe & 0,536 & 0,132 & 0,674 & 0,514 & 0,517 & 0,345 & 1,000 & & & & & & & & \\
\hline Temp & $-0,482$ & $-0,071$ & $-0,548$ & $-0,627$ & $-0,445$ & 0,448 & $-0,025$ & 1,000 & & & & & & & \\
\hline $\mathrm{HO}_{2} \mathrm{P}$ & $-0,326$ & $-0,140$ & $-0,366$ & $-0,472$ & $-0,490$ & 0,288 & $-0,098$ & 0,533 & 1,000 & & & & & & \\
\hline S Pom & 0,098 & 0,018 & 0,036 & $-0,020$ & $-0,104$ & 0,021 & 0,073 & $-0,025$ & 0,034 & 1,000 & & & & & \\
\hline S Chl & 0,092 & 0,276 & $-0,121$ & $-0,195$ & $-0,269$ & 0,322 & 0,118 & 0,216 & 0,017 & 0,680 & 1,000 & & & & \\
\hline S Phe & 0,097 & 0,040 & 0,009 & $-0,018$ & $-0,076$ & 0,175 & 0,138 & 0,027 & $-0,133$ & 0,829 & 0,772 & 1,000 & & & \\
\hline S Pro & 0,043 & 0,029 & $-0,005$ & $-0,046$ & $-0,114$ & 0,015 & 0,016 & 0,025 & 0,030 & 0,969 & 0,709 & 0,815 & 1,000 & & \\
\hline S Lip & 0,106 & 0,047 & 0,012 & $-0,035$ & $-0,123$ & 0,035 & 0,029 & 0,020 & 0,064 & 0,980 & 0,720 & 0,815 & 0,980 & 1,000 & \\
\hline S Car & 0,099 & 0,182 & $-0,016$ & $-0,052$ & $-0,166$ & 0,045 & 0,051 & $-0,036$ & 0,067 & 0,905 & 0,710 & 0,714 & 0,883 & 0,918 & 1,000 \\
\hline
\end{tabular}

entre elles, indépendants les uns des autres. Ainsi, à partir de 15 séries de 41 observations sur l'ensemble des deux sites, sept axes indépendants sont retenus en fonction de leur part respective de variance totale expliquée. Une nouvelle matrice est générée par les coordonnées des observations projetées sur les axes, résumant $95 \%$ de l'information de départ. Le taux de croissance $(G)$ n'est pas pris en compte dans les calculs de l'analyse en composantes principales mais projeté en variable illustrative (Conan et Comeau 1986). Ceci permet de préciser les périodes et sites de forte croissance.

Modèle de croissance

Les fluctuations de l'environnement sont intégrées par l'animal au niveau du poids sec et du taux de croissance; ainsi un modèle allométrique est utilisé, où l'exposant et le taux sont optimisés.

À la suite de Sebens (1982), les deux paramètres $G$ (taux de croissance) et $b$ (coefficient d'allométrie) du modèle

(1) $\frac{d W}{d t}=G \cdot W^{b}$

sont considérés respectivement comme fonction du milieu et de l'espèce. En intégrant à milieu constant $(G=\mathrm{cst}, b \neq 1)$ et après correction de la fraction journalière d'immersion $(\Delta t)$, on obtient :

(2) $W(t)=\left(W(t-1)^{c}+G(t) \cdot \Delta t \cdot c\right)^{1 / c}$

avec $t=1$ à $N(N=$ nombre d'observations) et $c=1-b$

Nous prenons comme hypothèse que la période de croissance est représentée par le temps de filtration égal à la durée d'immersion.

Le taux de croissance $G$ est expliqué par les axes principaux $X_{i}$ issus de 1'analyse factorielle. La relation est écrite :

(3) $\quad G(t)=\sum_{i} a_{i} X_{i}(t)+a_{\mathrm{o}}$

où $a_{i}$ représente la sensibilité de chaque coefficient.
Tableau 2. Pourcentage de variance expliquée par les sept axes principaux de l'Analyse en composantes principales.

\begin{tabular}{lcc}
\hline \hline Axes & \% Variance & Val. propres \\
\hline 1 & 35,007 & 5,25 \\
2 & 30,134 & 4,52 \\
3 & 11,752 & 1,76 \\
4 & 7,384 & 1,11 \\
5 & 4,933 & 0,74 \\
6 & 3,445 & 0,52 \\
7 & 2,197 & 0,33 \\
\hline
\end{tabular}

Le modèle est une extension de la régression orthogonalisée (Tomassone et al. 1983) à un cas de non-linéarité. Les paramètres sont estimés par optimisation à l'aide de l'algorithme du Simplex (Nelder et Mead 1965 ; Schnute 1982), basée sur la minimisation du critère qui représente l'erreur résiduelle (ER)

(4) $\quad \mathrm{ER}=\sum_{t}\left(W_{\mathrm{obs}}(t)-W_{\mathrm{cal}}(t)\right)^{2}$

La validité du modèle s'effectue par comparaison du critère à la somme des carrés totale (ST) en tenant compte du nombre de degrés de liberté de chaque terme.

(5) $S T=\sum_{t}\left(W_{\mathrm{obs}}(t)-\bar{W}\right)^{2}$, où $\bar{W}$ est la moyenne des $W_{\mathrm{obs}}(t)$

$$
F=\frac{(S T-\mathrm{ER}) / p}{\mathrm{ER} /(N-p)} \text { suit approximativement }
$$

une loi de Fisher $\mathrm{F}(p, N-p)$ où $p$ est le nombre de paramètres estimés et $N$ le nombre d'observations.

Les axes principaux $X_{i}$ étant indépendants, le calcul de leur contribution $c_{i}$ permet de les trier selon leur ordre d'importance dans l'explication du taux de croissance $G\left(c_{i}=a_{i}^{2} \lambda_{i}, \lambda_{i}\right.$ étant la nième valeur propre).

De nouvelles optimisations sont réalisées après le retrait successif des axes les moins importants. Deux modèles successifs sont comparés à l'aide du rapport : 

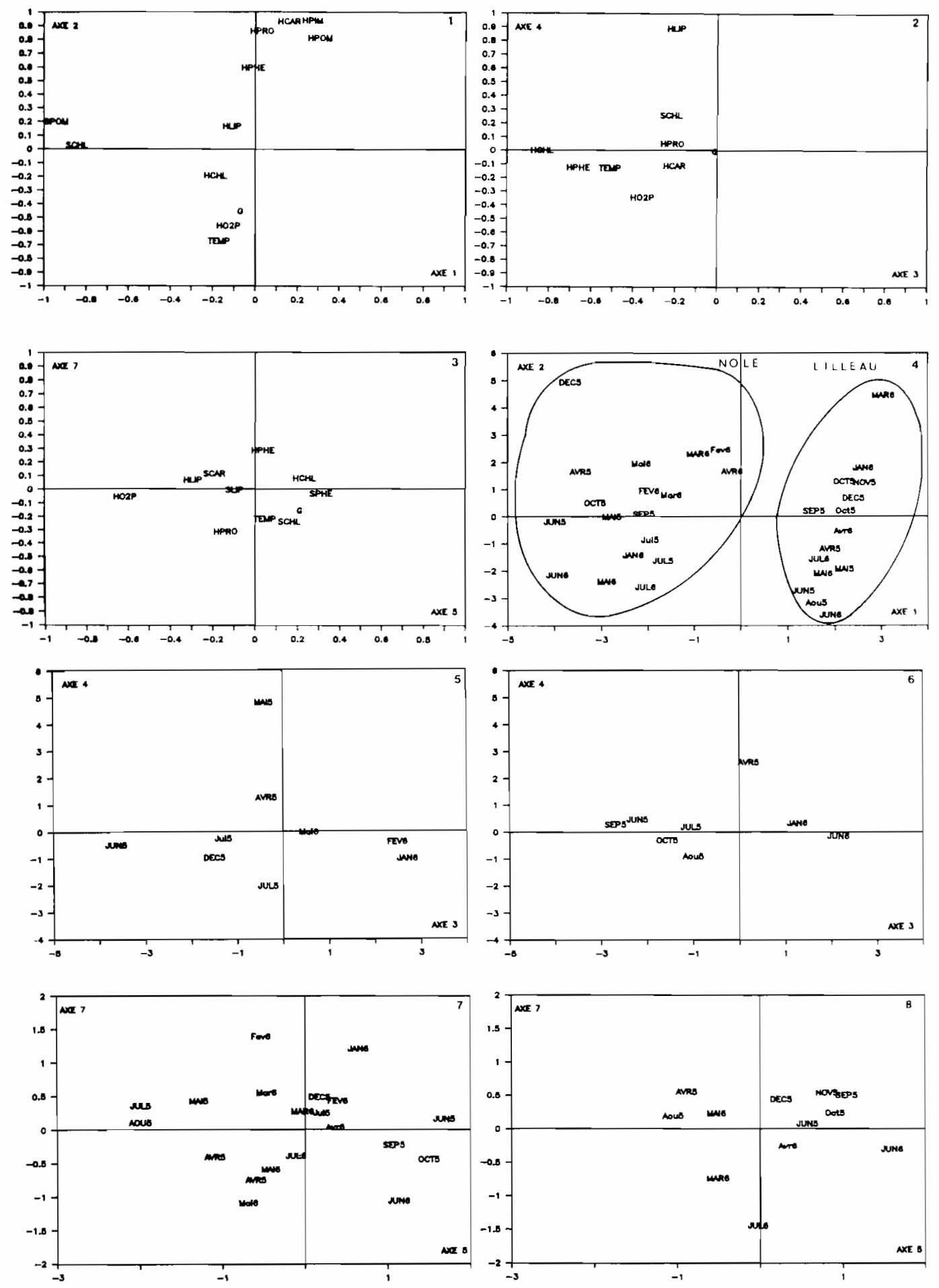

FIG. 2. Projection des descripteurs et observations sur les axes 1, 2, 3, 5 et 7 de l'Analyse en composantes principales (seuls les points bien représentés sont projetés). (1) à (3) : Corrélations entre les descripteurs et les axes. $G$ : Taux de croissance en variable illustrative projetée. Hydrologie : HPRO = protéines, HLIP = lipides, HCAR = carbohydrates, HPIM = seston minéral, HPOM = seston organique, HCHL = chlorophylle $a, \mathrm{HPHE}=$ phéopigments, TEMP = température, $\mathrm{HO}_{2} \mathrm{P}=$ pourcentage de saturation en oxygène. Sédiment $:$ SPOM $=$ matière organique, SCHL = chlorophylle $a, \mathrm{SPHE}=$ phéopigments, SPRO = protéines, SLIP = lipides, SCAR = carbohydrates. (4) à (8) : Projections des observations. Pour les axes 3, 4, 5 et 7 , chaque site est représenté séparément. Première classe sur le site de Nole : AVR5 = avril, MAI5 $=$ mai, JUN5 = juin, Jul5 = début Juillet, JUL5 = fin juillet, AOU5 = août, SEP5 = septembre, OCT5 = octobre 1985. Deuxième classe sur le site de Nole : DEC5 = décembre 1985, JAN6 = janvier, Fev6= début février, FEV6 = fin février, Mar6 = début mars, MAR6 = fin mars, Avr6 = début avril, AVR6 = fin avril, Mai6 = début mai, MAI6 = fin mai, JUN6 = juin, JUL6 = juillet 1986 . Première classe sur le site de Lilleau : AVR5 = avril, MAI5 = mai, JUN5 = juin, JUL5 = juillet, Aou5 = début août, AOU5 = fin août, SEP5 = septembre, Oct5 $=$ début octobre, OCT5 = fin octobre 1985. Deuxième classe sur le site de Lilleau : NOV5 = novembre, DEC5 $=$ décembre 1985 , JAN6 = janvier, Fev6 = début février, FEV6 = fin février, Mar6 = début mars, MAR6 = fin mars, Avr6 = début avril, AVR6 = fin avril, MAI6 = mai, JUN6 $=$ juin, JUL6 $=$ juillet 1986. 
TABlEaU 3. Corrélations entre les variables et les axes principaux de l'Analyse en composantes principales, ( $G$ : taux de croissance projeté en variable illustrative).

\begin{tabular}{|c|c|c|c|c|c|c|c|c|}
\hline Axes & & 1 & 2 & 3 & 4 & 5 & 6 & 7 \\
\hline $\begin{array}{l}\text { Variable } \\
\text { supplémentaire }\end{array}$ & $G$ & $-0,083$ & $-0,510$ & $-0,012$ & $-0,007$ & 0,210 & $-0,032$ & $-0,157$ \\
\hline $\begin{array}{l}\text { Variables } \\
\text { hydrologiques }\end{array}$ & $\begin{array}{l}\text { H Pro } \\
\text { H Lip } \\
\text { H Car } \\
\text { H Pim } \\
\text { H Pom } \\
\text { H Chl } \\
\text { H Phe } \\
\text { Temp. } \\
\mathrm{HO}_{2} \mathrm{P}\end{array}$ & $\begin{array}{r}0,030 \\
-0,109 \\
0,160 \\
0,229 \\
0,306 \\
-0,188 \\
-0,010 \\
-0,169 \\
-0,126\end{array}$ & $\begin{array}{r}0,862 \\
0,170 \\
0,932 \\
0,938 \\
0,847 \\
-0,190 \\
0,596 \\
-0,651 \\
-0,556\end{array}$ & $\begin{array}{r}-0,214 \\
-0,196 \\
-0,204 \\
0,044 \\
-0,008 \\
-0,834 \\
-0,662 \\
-0,511 \\
-0,356\end{array}$ & $\begin{array}{r}0,048 \\
0,891 \\
-0,112 \\
-0,129 \\
-0,130 \\
0,001 \\
-0,126 \\
-0,129 \\
-0,343\end{array}$ & $\begin{array}{r}-0,140 \\
-0,295 \\
-0,100 \\
-0,062 \\
0,038 \\
0,234 \\
0,037 \\
0,046 \\
-0,619\end{array}$ & $\begin{array}{r}0,219 \\
-0,140 \\
0,071 \\
-0,029 \\
-0,302 \\
0,333 \\
-0,194 \\
-0,437 \\
0,069\end{array}$ & $\begin{array}{r}0,312 \\
0,068 \\
0,029 \\
-0,083 \\
-0,054 \\
0,079 \\
0,285 \\
-0,218 \\
-0,056\end{array}$ \\
\hline $\begin{array}{l}\text { Variables } \\
\text { sédimentaires }\end{array}$ & $\begin{array}{l}\text { S Pom } \\
\text { S Chl } \\
\text { S Phe } \\
\text { S Pro } \\
\text { S Lip } \\
\text { S Car }\end{array}$ & $\begin{array}{l}-0,942 \\
-0,844 \\
-0,866 \\
-0,945 \\
-0,956 \\
-0,912\end{array}$ & $\begin{array}{l}0,199 \\
0,028 \\
0,197 \\
0,157 \\
0,173 \\
0,519\end{array}$ & $\begin{array}{r}0,134 \\
-0,220 \\
-0,003 \\
0,150 \\
0,120 \\
0,098\end{array}$ & $\begin{array}{r}-0,143 \\
0,257 \\
-0,020 \\
-0,119 \\
-0,112 \\
0,040\end{array}$ & $\begin{array}{r}-0,066 \\
0,161 \\
0,313 \\
-0,045 \\
-0,098 \\
-0,192\end{array}$ & $\begin{array}{r}-0,017 \\
0,027 \\
-0,016 \\
-0,071 \\
-0,020 \\
0,036\end{array}$ & $\begin{array}{r}0,087 \\
-0,238 \\
-0,034 \\
0,030 \\
-0,006 \\
0,113\end{array}$ \\
\hline
\end{tabular}

$$
F=\frac{\left(\mathrm{ER}_{2}-\mathrm{ER}_{1}\right) /\left(p_{1}-p_{2}\right)}{\mathrm{ER}_{1} / p_{1}}
$$

où $\mathrm{ER}_{i}$ désigne l'erreur résiduelle du modèle $i$ à $p_{i}$ paramètres. $F$ suit une loi de Fisher $\mathrm{F}\left(p_{1}, p_{2}\right)$. Tant que ce rapport n'est pas significatif, le modèle est considéré comme significatif. Comme la régression progressive pas à pas, dans un cas de linéarité, cette méthode permet de réduire le nombre d'axes significatifs.

Lorsque les paramètres du modèle sont déterminés, l'influence des descripteurs du milieu sur le poids final peut être estimée à travers une analyse de sensibilité à chaque coefficient $a_{i}$. Le tirage aléatoire d'une variable $T$ de moyenne nulle permet d'écrire :

$$
a_{i}^{\prime}=a_{i}(1+T),
$$

où $a_{i}^{\prime}$ est la valeur modifiée de $a_{i}$ introduite dans le modèle.

Si la distribution de $T$ suit une loi normale, le poids final suivra également une distribution normale dont la variabilité sera estimée à partir des résultats de 50 simulations. Le pourcentage de variation du poids final estimera la sensibilité du modèle à l'axe considéré, selon la classe et le site choisis.

Le modèle est ensuite appliqué pour comparer l'effet du site à conditions initiales semblables : poids initiaux de chaque classe ou temps d'immersion égaux. Le poids final exprime alors une croissance potentielle, fonction du temps d'immersion et du poids initial.

\section{Résultats}

\section{Analyse factorielle}

L'analyse en composantes principales, effectuée à partir du logiciel LADDAD (1985) permet de conserver 94,85\% de la variabilité sur les sept premiers axes (tableau 2) dont les trois premiers représentent à eux seuls $76,89 \%$.

Les projections des variables du milieu (fig. $2_{1-3}$ ) permettent d'identifier les corrélations avec les axes : l'axe 1 est représentatif de la totalité des fluctuations des variables sédimentaires (SPOM, SCHL, SPHE, SPRO, SLIP, SCAR) alors que l'axe 2 caractérise principalement l'évolution des variables hydrologiques hormis la chlorophylle $a$ et les lipides particulaires (tableau 3).

La projection du taux de croissance en tant que variable illustrative permet de montrer l'influence de la température sur les périodes de forte croissance. La projection des observations (fig. $2_{48}$ ) individualise les deux sites Nole et Lilleau, essentiellement par les caractéristiques sédimentaires très différentes. Le sédiment vaseux contient en moyenne $7 \%$ de matière organique pour $1 \%$ dans le sédiment sableux. Aux périodes de croissance, juillet à août 1985, mai à juillet 1986, s'oppose la période hivernale représentés par des amaigrissements marqués (mars 1986) et une nourriture potentielle détritique élevée (protéines et glucides particulaires) de qualité inférieure (fort seston minéral).

La comparaison des années 1985 et 1986 montre une répétition pour le site sableux de Lilleau, avec par ailleurs, une faible dispersion des observations caractérisant une stabilité du site. Le site vaseux de Nole montre une forte variabilité sur les axes 1 et 2 (décembre 1985, janvier 1986) avec une fluctuation interannuelle marquée (juin 1985 - juin 1986).

Les axes 3-4 (tableau 2) caractérisent les flux de nourriture avec les floraisons chlorophylliennes sur l'axe 3 en juin et septembre 1985 ainsi que les faibles quantités de chlorophylle en sortie d'hiver (janvier à mars 1986) (fig. $2_{2}, 2_{5}, 2_{6}$ ). Les floraisons phytoplanctoniques sont de faible amplitude pour les deux sites au printemps 1986 , de l'ordre de $3 \mu \mathrm{g} \cdot \mathrm{L}^{-1}(10$ $\mu \mathrm{g} \cdot \mathrm{L}^{-1}$ en 1985), d'où l'absence de corrélation avec les axes 3 et 4 . Le site de Lilleau a la particularité de présenter une floraison automnale marquée en septembre $1985\left(7 \mu \mathrm{g} \cdot \mathrm{L}^{-1}\right)$ qui n'est pas observée dans la partie centrale du bassin.

Les lipides particulaires, constituant une nourriture potentielle énergétique, sont associés à l'axe 4 qui oppose une période déficitaire en été (Nole, août 1985) à de fortes valeurs au printemps (Lilleau, avril 1985 ; Nole, mai 1985).

La saturation en oxygène est la variable la plus corrélée à l'axe 5, sur lequel les observations de période estivale, consécutives aux floraisons phytoplanctoniques, sont présentes (fig. $2_{7}$ et $2_{8}$ ). La période automnale se situe à l'opposé sur cet axe, associé à des chutes de saturation en oxygène et à de fortes teneurs en phéopigments dans le sédiment. Ces deux variables 

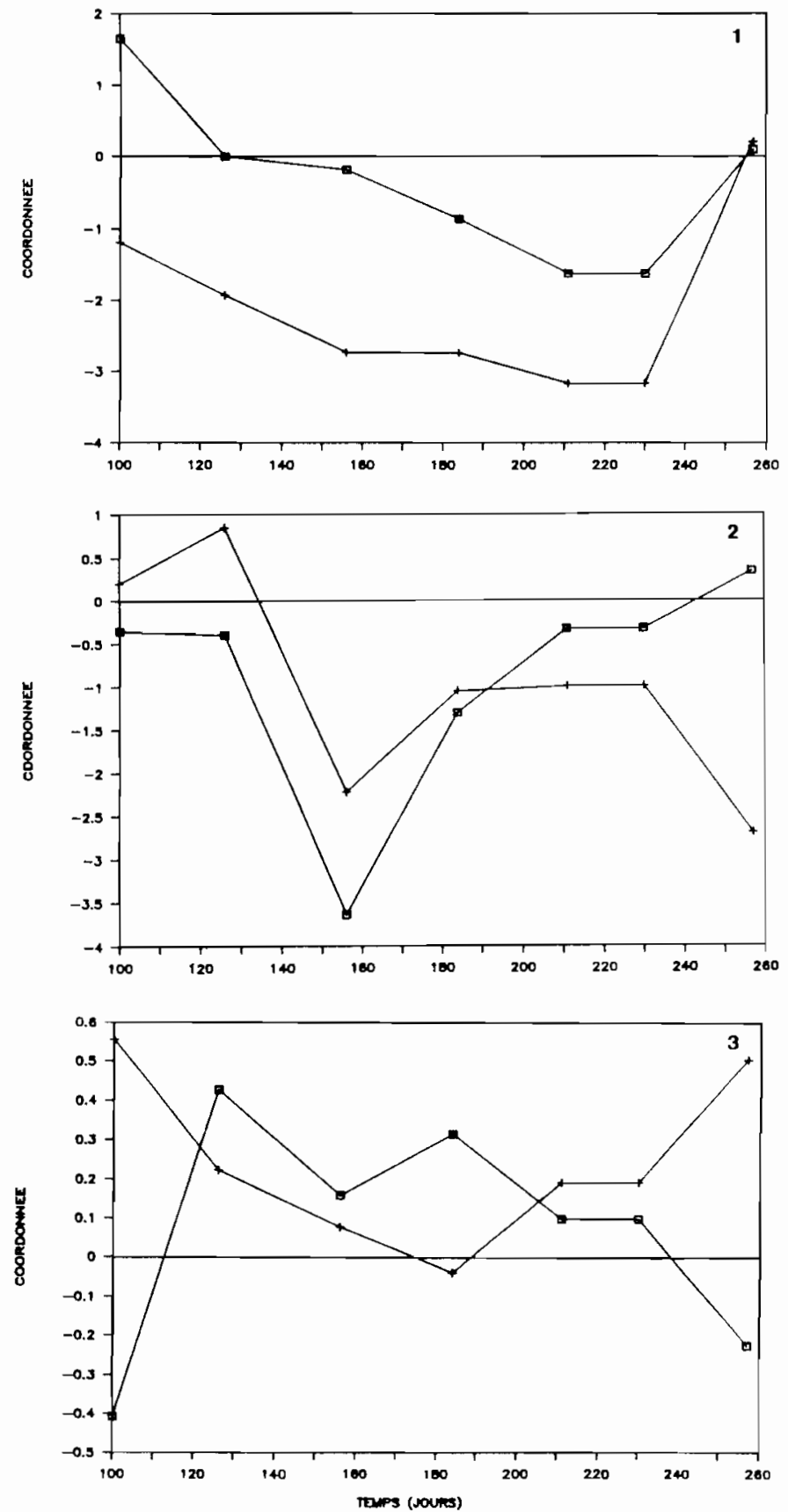

FIG. 3. Analyse en composantes principales. (1) à (3) : évolution des axes 2,3 et 7 en fonction du temps. Les particularités des sites de Nole $(\square)$ et de Lilleau $(+)$ pour la première période de croissance (mars-octobre) sont analysées : axe 2 , différence systématique des teneurs en PIM et POM sur les deux sites ; axe 3, floraisons phytoplanctoniques comparables au printemps sur les deux sites et absente en automne sur le site de Nole (valeurs négatives des coordonnées); axe 7, opposition des teneurs en protéines faibles sur Nole et fortes sur Lilleau, aux périodes de floraisons phytoplanctoniques printanière et automnale.

indiquent une dégradation du phytobenthos dans le milieu. L'axe 5 représente par ailleurs le deuxième axe de projection du taux de croissance. L'axe 7 est représenté par les variables hydrologiques, la température, les protéines et les phéopigments, précisant des apports de nourriture ponctuels (avril, mai et juillet 1986). Les axes secondaires 5 et 7 (fig. $2_{3}$ ) sont donc liés à des fluctuations saisonnières ponctuelles. Sur
TABlEAU 4. Contributions des six axes retenus, explicatifs du modèle de croissance $\left(a_{0}=4,968, \mathrm{~b}=1,735\right)$.

\begin{tabular}{lcr}
\hline \hline Axes & $\%$ Variance & $a_{i}$ \\
\hline 2 & 58,30 & $-3,087$ \\
7 & 18,17 & $-6,383$ \\
4 & 11,90 & 2,818 \\
5 & 9,28 & 3,0448 \\
3 & 1,23 & 0,7178 \\
1 & 1,11 & $-0,3961$ \\
& ER $=19,85 \quad$ ST $=166$ & \\
& $n=37 \quad p=8$ & \\
& $\mathrm{~F}(8,37)=26,46$ & \\
\hline
\end{tabular}
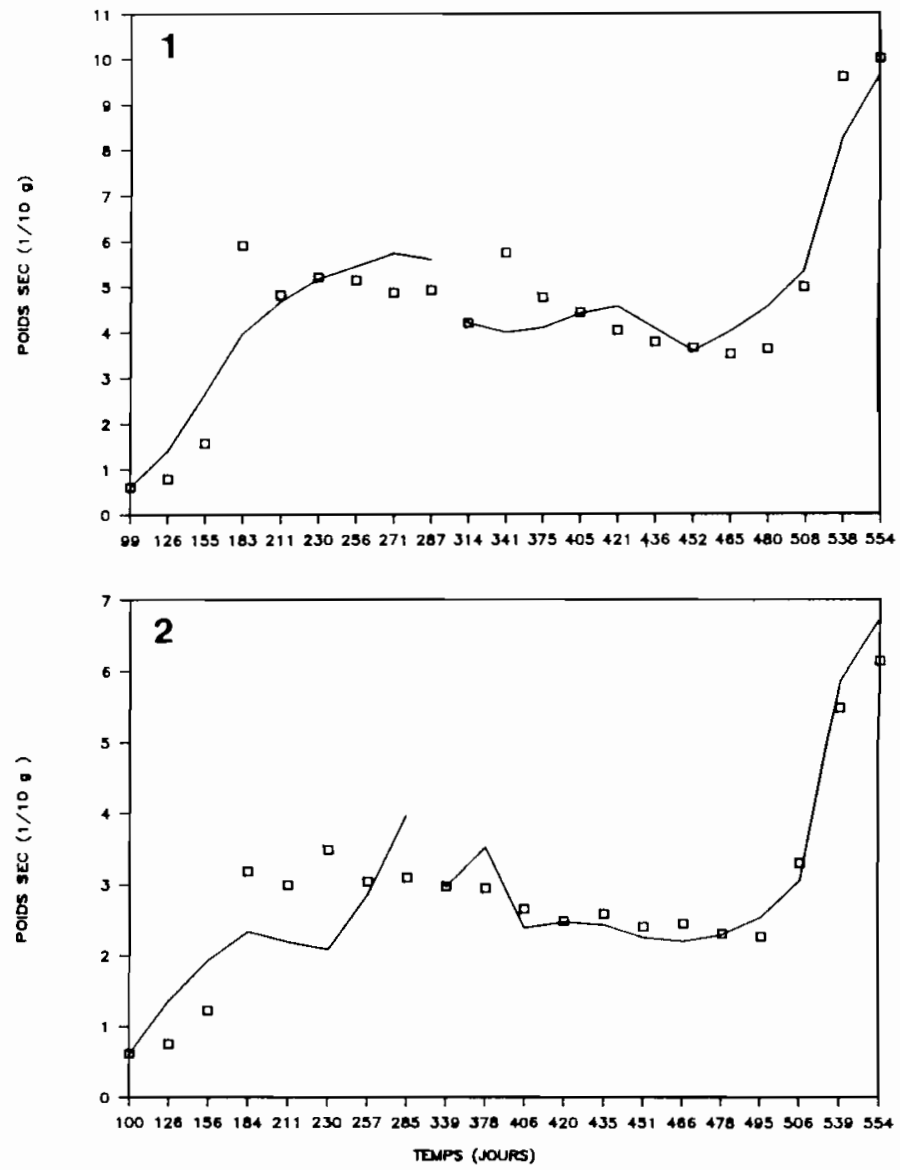

FIG. 4. Modèle de croissance fonction des axes principaux de l'ACP (ligne continue) et observations (ㅁ) pour les deux classes sur le site de Lilleau (1) et le site de Nole (2). Les axes 1, 2, 3, 4, 5 et 7 ont été sélectionnés d'après leur contribution à l'explication du taux de croissance.

l'axe 6 (tableau 3) on trouve également des apports ponctuels de nourriture (chlorophylle $a$ ) avec, parallèlement, une température élevée (septembre et octobre 1985). Les coordonnées des observations sur les axes 2,3 et 7 sont projetées en fonction du temps et illustrent la différence entre sites vaseux et sableux (fig. 3).

Modèle de croissance

Les sept axes résultant de l'analyse factorielle identifient de nouveaux descripteurs synthétiques du milieu utilisés par le modèle de croissance. Un premier modèle est conçu avec les 7 
TABLEAU 5. Sensibilité du poids final aux coefficients de régression des six axes retenus (valeurs absolues et pourcentage).

\begin{tabular}{|c|c|c|c|c|c|c|c|c|}
\hline \multirow[b]{3}{*}{ Site } & \multicolumn{4}{|c|}{$1^{\mathrm{rc}}$ classe } & \multicolumn{4}{|c|}{$2^{e}$ classe } \\
\hline & \multicolumn{2}{|c|}{ Nole } & \multicolumn{2}{|c|}{ Lilleau } & \multicolumn{2}{|c|}{ Nole } & \multicolumn{2}{|c|}{ Lilleau } \\
\hline & Val. abs. & $\%$ & Val. abs. & $\%$ & Val. abs. & $\%$ & Val. abs. & $\%$ \\
\hline Axes & & & & & & & & \\
\hline 1 & 0,560 & 25,0 & 0,476 & 7,28 & 0,570 & 17,99 & 0,967 & 20,66 \\
\hline 2 & 0,905 & 40,36 & 4,206 & 64,26 & 0,332 & 10,49 & 1,105 & 23,58 \\
\hline 3 & 0,388 & 17,34 & 0,661 & 10,10 & 0,669 & 21,14 & 0,444 & 9,47 \\
\hline 4 & 0,147 & 6,57 & 0,002 & 0,03 & 0,532 & 16,78 & 0,077 & 1,65 \\
\hline 5 & 0,007 & 0,32 & 0,219 & 3,33 & 0,309 & 9,76 & 0,778 & 16,61 \\
\hline 7 & 0,233 & 10,41 & 0,982 & 15,00 & 0,755 & 23,84 & 1,313 & 28,03 \\
\hline
\end{tabular}

\section{SENSIBILITE}

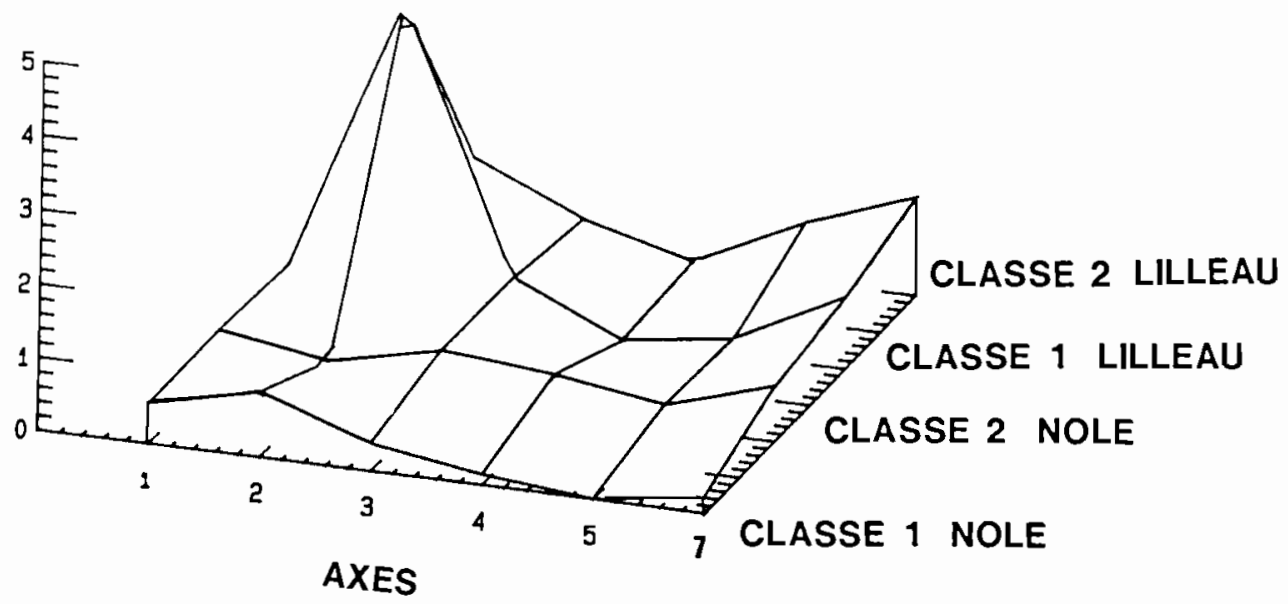

Fig. 5. Analyse de sensibilité à des fluctuations aléatoires des axes $1,2,3,4$, 5, et 7 retenus dans le modèle sur les sites de Nole et de Lilleau. La classe d'âge 1 est sensible à l'axe 2 (variables hydrologiques PIM, POM). La deuxième classe est sensible à l'axe 7 (protéines et phéopigments de l'eau).

axes, dont les contributions sont ensuite calculées. Les axes les moins importants en termes de contribution sont retirés un à un du modèle jusqu'à ce que la perte d'information du nouveau modèle soit significative. Les différentes simulations effectuées et la comparaison des critères obtenus (voir équation (4)) permettent de retenir finalement six axes principaux explicatifs de la croissance (tableau 4). Les contributions des axes retenus sont classées par ordre décroissant. L'axe 1 , représentatif des fluctuations sédimentaires, ne peut être enlevé sans augmentation notable du critère. Les conditions hydrologiques (axe 2) constituent le principal descripteur du milieu explicatif de la croissance $(58,3 \%)$. Il est à noter que le test du bâton brisé et la comparaison des valeurs propres par rapports à 1 (Legendre et Legendre 1979) indiquent que les axes d'ordre supérieur à 4 expliquent une part négligeable de la variance totale. Cependant, l'importance prise par l'axe 7 dans le modèle (tableau 4) et son identification à des événements écologiques ponctuels montrent qu'il ne s'agit pas d'un simple bruit de l'échantillonnage.

Les optimisations et les observations sont présentées sur la figure 4. Les performances de croissance observées sont supérieures en classe 1 et 2 sur le site de Lilleau dans le nord du bassin. L'optimisation de la classe 1 du site de Nole présente des valeurs inférieures aux observations. Par ailleurs sur le site 2, deux écarts sont observés par rapport aux observations : au mois de juillet 1985 , du fait d'une ponte partielle notable qui a suivi cette date, ainsi qu'au mois de décembre 1985 .

L'analyse de sensibilité permet d'obtenir des informations complémentaires quant à l'importance de chaque descripteur sur la croissance des palourdes en fonction du site et des classes d'âge (tableau 5, fig. 5).

Les paramètres hydrologiques de l'axe 2 sont responsables principalement de la sensibilité de la classe 1 sur les 2 sites avec $40,36 \%$ (Nole) et $64,24 \%$ (Lilleau). Par contre, la sensibilité de la classe 2 est expliquée par l'axe 7 (Nole, 23,84\%; Lilleau, $28,03 \%$ ).

Par ailleurs des différences de sensibilité apparaissent selon les années et les sites et proviennent des fluctuations interannuelles et spatiales (respectivement). L'axe 1 représentant les variables sédimentaires est globalement plus important sur Nole $(25 \%, 18 \%)$ que sur Lilleau $(7,3 \%$ et $21 \%)$.

\section{Simulations}

En raison des écarts de poids initiaux importants, observés pour la $2^{\mathrm{e}}$ classe, suite à une croissance et à un effort de repro- 

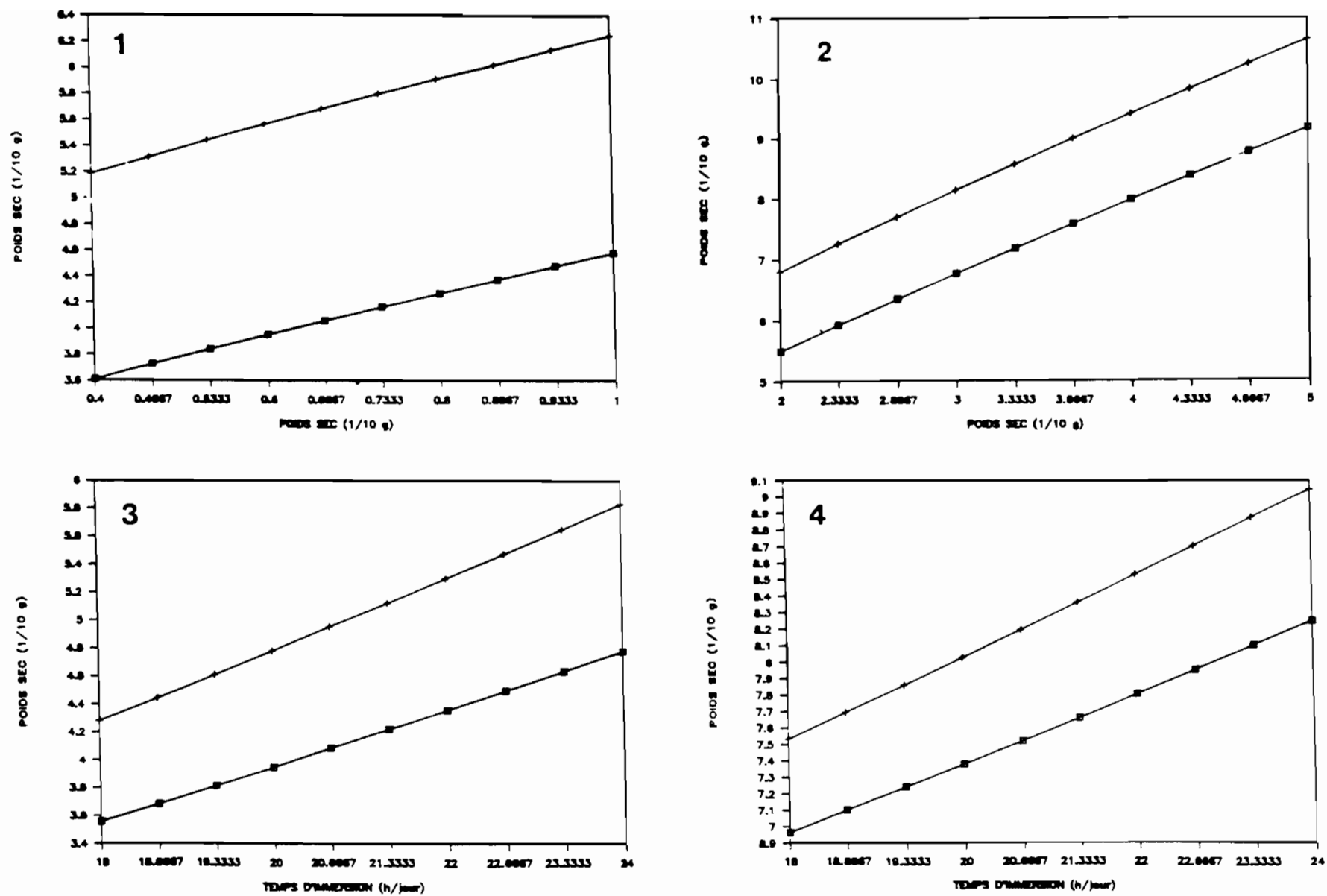

FIG. 6. Simulation de la croissance potentielle $(1 / 10 \mathrm{~g})$ sur les sites de Nole $(\square)$ et de Lilleau $(+): 1)$ en fonction du poids initial $(1 / 10 \mathrm{~g})$ pour les classes 1 (1) et $2(2) ; 2$ ) en fonction du temps d'immersion (h/d) pour les classes 1 (3) et 2 (4). Pour un même poids initial ou un même temps d'immersion, le site de Lilleau présente toujours une croissance supérieure.

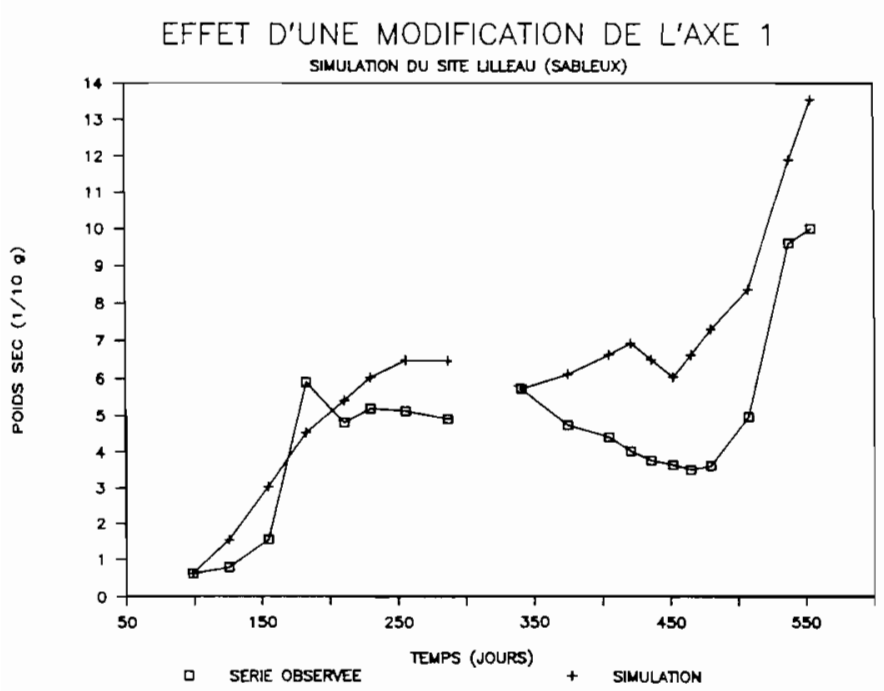

FIG. 7. Simulation de la croissance potentielle $(1 / 10 \mathrm{~g})$ du site de Lilleau avec modification de l'axe $1(+)$ et observations $(\square)$. La différence de sensibilité des sites vaseux et sableux à l'axel (sédiment) s'explique par l'effet inhibiteur lié à la remise en suspension.

duction différents entre les sites, aucune information directe ne peut découler des observations. Les simulations à poids initial ou à temps d'immersion constant (fig. 6) conservent les mêmes
EFFET D'UNE MODIFICATION DES AXES 2,3,7

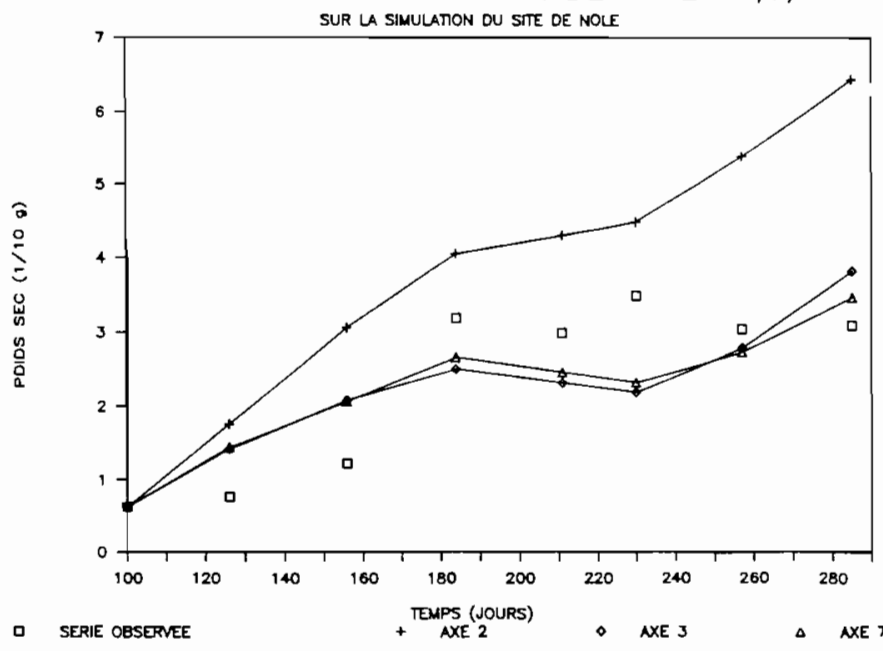

FIG. 8. Simulation de la croissance potentielle $(1 / 10 \mathrm{~g})$ du site de Nole avec modification de l'axe $2(+), 3(\diamond), 7(\triangle)$, du site de Lilleau et observations ( $\square$ ).

tendances par rapport aux observations : les croissances des palourdes sur le site de Lilleau sont toujours supérieures.

Une simulation réalisée sur le site de Lilleau en utilisant les coordonnées des paramètres sédimentaires du site vaseux (axe 1) indépendamment des caractéristiques hydrologiques, tend à 
montrer que le facteur «quantité de nourriture» du sédiment n'est pas seul à intervenir (fig. 7). En effet la croissance est fortement favorisée dans le cas présent, en opposition avec les observations du site de Nole. Par ailleurs, des simulations au cours desquelles les valeurs des axes, 2,3 et 7 du site 2 ont été substituées à celles des mêmes axes du site 1 (successivement), pendant la première période de croissance (1985), ont été effectuées (fig. 8).

\section{Discussion}

\section{Classification des descripteurs}

Les corrélations observées entre les populations de bivalves, la colonne d'eau et l'interface eau-sédiment nécessitent d'être confirmées par des études pluri-annuelles afin de vérifier l'influence de chaque variable et d'éviter des corrélations accidentelles (Héral et al. 1984). Cette étude a permis, par l'utilisation du modèle de croissance, de mettre en évidence les principales variables environnementales agissant sur les fluctuations du poids sec de l'animal. Les périodes de croissance sont associées à de fortes températures, teneurs en chlorophylle, lipides et protéines dans l'eau, alors que les périodes d'amaigrissement sont observées à faible température, forte turbidité et une teneur en nourriture détritique élevée. L'effet de la température a été démontré par de nombreux auteurs, en particulier sur Ruditapes philippinarum par Mann et Glomb (1978) et Mann (1979). Les taux de filtration et de respiration sont modifiés par la température selon Bernard (1983) et Laing et al. (1987). Par ailleurs, le contenu lipidique de la nourriture phytoplanctonique influence la croissance de la palourde japonaise selon Laing et al. (1987). Si les principaux descripteurs sont identiques au cours des deux années sur le site, la sensibilité dépend du niveau trophique (floraison phytoplanctonique). Par ailleurs, les conditions hivernales en classe 2, marquées par des remises en suspension de sédiment plus fréquentes, peuvent expliquer les différences de sensibilité.

\section{Effet du site}

L'effet du site est visualisé par les fortes différences de croissance en observation et simulations (fig. 4). Les palourdes localisées dans le nord du bassin présentent une croissance systématiquement plus élevée que la population située dans la partie médiane du bassin. Cet effet peut être dû : 1) à la différence dans le temps d'immersion moyen correspondant à un temps de filtration potentiel, 2) à la quantité de nourriture disponible dans la colonne d'eau et le sédiment, et 3 ) à la nature physique du sédiment, associée à la vitesse du courant.

Le temps d'immersion est considéré comme un facteur important en zone intertidale dans le contrôle de la croissance comme l'ont démontré Glock et Chew (1979) sur $R$. philippinarum, Griffiths (1981) sur Choromytilus meridionalis, Gillmor (1982) sur différents bivalves, et Goulletquer et Bacher (1989) sur $R$. philippinarum. Hormis le temps de filtration, l'émersion a des répercussions sur la physiologie de l'animal (Bayne et al. 1976 ; Fang 1982). Les simulations effectuées à temps d'immersion constants n'inversent pas la tendance mais diminuent les écarts observés entre les deux sites (fig. 6). Le temps d'immersion intervient par conséquent sur les performances finales de croissance sans être le principal facteur explicatif des différences observées.

Les déplacements des masses d'eau dans le bassin de Marennes-Oléron, ainsi que la biomasse en présence de cultures d'huîtres $C$. gigas, 82000 t en 1985 (Bacher et al. 1986), interviennent sur la disponibilité en nourriture au niveau quantitatif et qualitatif entre la partie nord et médiane du bassin (Héral 1987 ; Bacher, com. pers.). Une déplétion de nourriture, occasionnée par l'activité de filtration des bivalves suspensivores est suggérée par certain auteurs : Fréchette et Bourget (1985a) montrent que la déplétion en matériel organique particulaire limite la croissance de Mytilus edulis. De même, Cloern (1982) et Cohen et al. (1984) suggèrent que les organismes benthiques sont présents en quantité suffisante pour réduire la biomasse phytoplanctonique dans les sites étudiés. Peterson et Black (1987) démontrent que la déplétion de nourriture dans la colonne d'eau constitue un effet prioritaire sur la croissance des suspensivores. Au cours de cette étude, la floraison phytoplanctonique automnale (axes 3-4, de l'ACP) est observée uniquement dans la partie nord du bassin. La biomasse phytoplanctonique se développant dans la partie médiane du bassin est de faible importance en raison d'une forte turbidité générale de la zone estuarienne (Héral et al. 1983). Du fait de la circulation générale nord-sud et du temps de résidence des eaux, il est probable que les floraisons phytoplanctoniques de moindre intensité sont consommées lors des passages successifs sur les élevages d'huîtres, avant d'atteindre le site de Nole. Par ailleurs, le contenu supérieur en matière organique au niveau du sédiment vaseux ne semble pas constituer un effet positif sur la croissance malgré la sensibilité des populations au descripteur 1 (fig. 5).

Selon Wildish et Kristmanson (1979), le couplage des facteurs vitesse du courant et rugosité du sédiment, régule la croissance des suspensivores avec une inhibition à des vitesses supérieures à $30 \mathrm{~cm} \cdot \mathrm{s}^{-1}$. La forte variabilité de la vitesse du courant sur le site de Nole, qui peut atteindre des valeurs instantanées supérieures à $1 \mathrm{~m} \cdot \mathrm{s}^{-1}$, faciliterait les remises en suspension ponctuelles. Si celles-ci sont facilitées par l'action hydrodynamique sur les sédiments vaseux par rapport au sédiment sableux (Jonge et van den Bergs 1987), la qualité nutritive de la nourriture (axe 2), définie par le rapport minéral-organique est affectée (Berg et Newell 1986 ; Bayne et al. 1987).

Par ailleurs Fréchette et Bourget (1985b) associent la matière organique particulaire remise en suspension près des zones d'élevage de $M$. edulis, principalement au phytoplancton filtré et rejeté sous forme de pseudofèces ou de fèces dues à une digestion partielle de qualité nutritive inférieure. Dans la partie médiane du bassin de Marennes-Oléron, Sornin et al. (1983) montrent également l'effet des biodépôts sur le contenu organique du sédiment ainsi que leur remise en suspension (Sornin et al. 1986). Ainsi à la différence de Baillie et Welsh (1980), la remise en suspension de l'interface eau-sédiment ne constitue pas nécessairement un avantage pour les filtreurs du fait de la qualité nutritive de la nourriture, en particulier de la surcharge minérale, qui peut occasionner des croissances négatives (Vahl 1980 ; Héral 1987). La simulation réalisée sur le site de Lilleau (fig. 7) tend à prouver que le seston minéral remis en suspension représente un facteur limitant de la croissance de Ruditapes philippinarum comme l'indiquent Daou et Goulletquer (1988). Toutefois, aucune corrélation significative entre le contenu minéral de l'eau et les paramètres du sédiment n'est observée (tableau 1). Ceci peut s'expliquer par la rapidité des processus au cours des remises en suspension (Jonge et van den Bergs 1987), phénomènes non décelables avec le pas de temps semi-mensuel adopté.

\section{Sensibilité du modèle à la stratégie d'échantillonnage}

Le modèle montre certaines lacunes dans l'explication de la 
croissance sur les deux sites en 1985 ( $1^{\text {re }}$ classe). Sur le site sableux une croissance très rapide est observée aux mois d'avril et de mai, suivie d'une ponte partielle non expliquée par le modèle. La croissance observée sur le site vaseux est plus régulière que la première. Le modèle a toutefois tendance à lisser la croissance en la surestimant légèrement au début du printemps, en l'affaiblissant de mai à juin pour l'accélérer considérablement en août.

Les différences obtenues en 1985 sont dues à une mauvaise représentation des relations trophiques ou bien du milieu. Ceci pose deux problèmes : celui de la formulation du modèle et de la limite de son pouvoir explicatif ou prédictif, et celui du suivi du milieu, c.-à-d. des variables choisies et du plan d'échantillonnage. La première catégorie de limitations de la méthodologie définie comprend l'existence possible d'effets seuils (création de pseudofèces par exemple), de déphasages (mécanismes de régulation de la filtration ou de l'assimilation) ou interactions non prises en compte. Dans la deuxième, en dehors des variables non suivies, un défaut d'explication peut provenir des biais d'échantillonnage ou du pas de temps retenu. La modélisation apparaît conforme aux observations en 1986. La méthode d'analyse a également été appliquée de façon satisfaisante en 1984 et 1985 (Goulletquer et Bacher 1989). On peut alors raisonnablement penser que le manque d'adéquation trouvé en 1985 vient d'un défaut du plan d'échantillonnage.

Des différences notables sont à rechercher dans les caractéristiques hydrologiques de chaque site. Les projections des observations sur les axes 2, 3 et 7 de l'ACP illustrent les particularités propres aux sites sableux et vaseux au cours de la première période de croissance (1985) (fig. 3). Le modèle est sensible principalement à ces 3 axes $(68 \%$ et $90 \%$ de la sensibilité totale pour les sites 1 et 2 respectivement. Pour la période de début de croissance (mars à juin), on observe des différences systématiques entre les sites. La prise en compte de l'ordre de grandeur de la différence entre les projections et du coefficient de régression $a_{i}$ de l'axe numéro $i$, incite à penser que l'axe 2 est responsable des différences de croissance $\left(\Delta \sim 2, a_{2}=-3\right)$. D'après les signes de $\Delta$ et $a_{i}$, on note que l'axe 2 a un effet inhibiteur sur la croissance sur site vaseux $\left(\Delta \cdot a_{2}<0\right)$. Les modifications des axes 2,3 , 4 et 7 du site 1 confirment l'importance de l'axe 2 (fig. 8 ). Les résultats de l'ACP ont montré que l'axe 2 est lié positivement aux seston, protides, carbohydrates et température. Le retour aux données d'origine fait ressortir le lien entre les fortes valeurs des projections sur l'axe 2 et les valeurs, également fortes, du seston minéral pendant les mois de mai et de juin sur le site vaseux. L'importance prise ainsi par le seston minéral dans la sensibilité du modèle à des périodes cruciales de croissance et sur un site vaseux appelle des remarques sur la méthodologie employée. Il existe peutêtre un biais des estimateurs de certaines variables hydrologiques. Une précision trop faible peut également générer des artefacts numériques dans le traitement non linéaire des données. Deux directions de recherche sont a priori envisageables : explication de la variabilité de certains paramètres hydrologiques et amélioration des estimateurs pour l'utilisation d'un pas de temps d'échantillonnage inférieur au mois.

Héral (1987) a déjà montré, sur un site vaseux, la corrélation existant entre la concentration particulaire et l'effet de la houle. Ward et al. (1984), Ward (1985) et Demers et al. (1987) ont mis en évidence l'effet du vent sur les concentrations particulaires dans des zones estuariennes de faible profondeur (entre 1 et $6 \mathrm{~m}$ ). Force du vent, hauteur d'eau et rugosité du fond sont les paramètres contrôlant l'intensité de la remise en suspension.
D'après ces mêmes auteurs, l'effet du vent est inférieur à celui du courant de marée jusqu'à un certain seuil de force du vent, au-delà duquel le premier devient prépondérant.

Dans une région à regime de veut irrégulier, une variabilité importante de certains paramètres hydrologiques peut donc se produire à une échelle de temps de l'ordre de quelques jours. Ainsi l'impact sur les mollusques ne peut être négligé à cette échelle, comme le suggèrent Fréchette et Bourget (1987), et impose une diminution du pas d'échantillonnage. Cette diminution aura pour conséquence d'améliorer le calcul de la réponse des mollusques aux variations du milieu. L'augmentation consécutive de l'effort d'échantillonnage sera compensée par un allégement en période d'amaigrissement hivernal, où les données de croissance sont relativement stables sur chacun des sites suivis. Enfin une loi empirique reliant la concentration particulaire à l'intensité du vent doit permettre de reconstituer le cycle du seston minéral à partir du régime de vent journalier, d'estimer et de corriger un biais du plan d'échantillonnage précédemment défini.

\section{Remerciements}

Les auteurs remercient M. P. Gros pour ses critiques, M. M. Héral et les arbitres anonymes pour leur aide constructive à la rédaction de la publication, ainsi que Mlle S. Taillade pour la frappe de ce manuscrit.

\section{Références}

Bacher, C., J. P. Baud, A. Bodoy, J. M. Deslous-Paoli, J. P. Dréno, M Héral, D. Maurer et J. Prou. 1986. A methodology for the stocks assessments of cultivated oysters along the French Atlantic coasts. ICES, CM 1986/K $36: 14$ p.

Ballue, P. W. ET B. L. WELSh. 1980. The effect of tidal resuspension on the distribution of intertidal epipelic algae in an estuary. Estuar. Coastl. and Mar. Sci. $10: 165-180$.

Bayne, B. L., R. J. Thompson et J. Widdows. 1986. 1. Physiology, p. 121206. Dans B. L. Bayne [éd.] Marine Mussels, their ecology and physiology. Cambridge University Press, Cambridge.

BAYNE, B. L. ET J. WIDDows. 1978. The physiological ecology of two populations of Mytilus edulis L. Oecologia 37 : 137-162.

Bayne, B. L., A. J. S. Hawkins ET E. NavarRo. 1987. Feeding and digestion by the mussel Mytilus edulis L. (Bivalvia : Mollusca) in mixtures of silt and algal cells at low concentrations. J. Exp. Mar. Biol. Ecol. 111 : 122.

Berg, J. A. et R. I. E. Newell. 1986. Temporal and spatial variations in the composition of seston available to the suspension feeder Crassostrea virginica. Estuar. Coast. Shelf Sci. $23: 375-386$.

Bernard, F. R. 1983. Physiology and the mariculture of some northeastern Pacific Bivalve Molluscs. Can. Spec. Publ. Fish. Aquat. Sci. $63: 24$ p.

Bodoy, A. ET M. R. Plante-CuNY. 1984. Relation entre l'évolution saisonnière des populations de palourdes Ruditapes decussatus et celles des microphytes benthiques et planctoniques (Golfe de Fos, France). Haliotis $14: 71-78$.

Cloern, J. E. 1982. Does the benthos control phytoplankton biomass in South San Francisco Bay? Mar. Ecol. Progr. Ser. $9: 191-202$.

Cohen, R. H., P. V. Dresler, E. J. P. Phillips et R. L. Cory. 1984. The effect of the asiatic clam, Corbicula fluminea on phytoplankton of the Potomac River, Maryland. Limnol. Oceanogr. 29 : 170-180.

Conan, G. Y. ET M. ComeaU. 1986. Functional maturity and terminal molt of male snow crab, Chionoecetes opilio. Can. J. Fish. Aquat. Sci. 43 : 17101719.

DaOU, R., et P. GoulletQueR. 1988. Effets de la turbidité sur les palourdes adultes Ruditapes philippinarum (Adams et Reeve) : croissance, mortalité, effort de reproduction, composition biochimique. Océanis $14: 375-389$.

Demers, S., J. C. Therriault, E. Bourget Et A. Bah. 1987. Resuspension in the shallow sublittoral zone of a macrotidal estuarine environment: wind influence. Limnol. Oceanogr. $32: 327-339$.

FANG, Y. 1982. Tidal zonation and cardiac physiology in four species of bivalves from Hong-Kong, p. 849-858. Dans N. S. Morton et C. K. Tseng [éd.] Proceedings of the First International Marine Biological Workshop: The 
Marine Flora and Fauna of Hong-Kong and Southern China. Hong-Kong 1980. Hong-Kong University Press, Hong-Kong.

FrÉCHETTE, M. ET E. BouRGET. 1985a. Food limited growth of Mytilus edulis L. in relation to the benthic boundary layer. Can. J. Fish. Aquat. Sci. 42: $1166-1170$.

1985b. Energy flow between the pelagic and henthic zones: Factors controlling particulate organic matter available to an intertidal mussel bed. Can. J. Fish. Aquat. Sci. 42 : 1158-1165.

1987. Significance of small-scale spatiotemporal heterogeneity in phytoplankton abundance for energy flow in Mytilus edulis. Mar. Biol. $94: 231-240$

GLLmOR, R. B. 1982. Assessment of intertidal growth and capacity adaptations in suspension feeding bivalves. Mar. Biol. 68 : 277-286.

GLOCK, J. W. ET K. K. CHEW. 1979. Growth, recovery and movement of Manila clams, Venerupis japonica (Deshayes) at Squaxin island Washington. Proceed. Natl. Shellfish Assoc. $69:$ 15-20.

GOULlETQuer, P. ET C. BACHER. 1989. Empirical modelling of the growth of $R$. philippinarum by means of non-linear regression on factorial coordinates. Aquatic Living Resources (sous presse).

Goulletquer, P., M. Nedhif et M. Héral. 1989. Ptoduction de palourdes japonaises Ruditapes philippinarum (Adams et Reeve) en bassin semifermé : approche énergétique et relations trophiques. Aquaculture 73 (sous presse).

Griffiths, R. J. 1981. Population dynamics and growth of the bivalve Choromytilus meridionalis $(\mathrm{Kr})$ at different tidal levels. Estuar. Coast. Shelf Sci. $12: 101-118$.

Héral, M., D. Razet, J. M. Deslous-Paoli, J. P. Berthomé et J. Garnier. 1983. Caractéristiques saisonnières de l'hydrologie du complexe estuarien de Marennes-Oléron (France). Rev. Trav. Inst. Pêches Marit. 46:97-119.

Héral, M., J. J. Deslous-Paoli, D. Razet et J. Prou. 1984. Essai de mise en évidence in situ de paramètres biotiques et abiotiques de l'eau et de l'interface eau-sédiment intervenant dans la population de l'huître Crassostrea gigas. Océanis $10: 465-475$.

HéRAL, M. 1987. Evaluation of the carrying capacity of Molluscan Shellfish Ecosystems. Shellfish culture Development and Management. Colloque international à La Rochelle (France), 4-9 mars 1985. Ed. IFREMER : 297-318.

Héral, M., J. M. Deslous-Paoli, J. Prou et D. Razet. 1987. Relations entre la nourriture disponible et la production des mollusques en milieu estuarien : variabilité temporelle de la colonne d'eau. Haliotis $16: 149-158$.

Héral, M., P. Goulletquer, B. Ravail, J. Prou, D. Razet et S. Y. MaesTRINI. 1988. Difficulties in sampling for estimation of phytoplankton biomass in estuarine conditions: variabilities and precisions at different temporal scales, in the case of Marennes-Oléron. Group for Aquatic Primary Productivity (G.A.P.), atelier international, avril 1988, CREMA L'Houmeau, France.

JONGE, V. N. DE ET J. VAN DEN BERGS. 1987. Experiments on the resuspension of estuarine sediments containing benthic diatoms. Estuar. Coastl. Shelf Sci. $24: 725-740$.

LADDAD. 1985. Manuel de référence ADDAD. Éd. Addad, Paris. 150 p.

LAING, I., S. D. UTTING ET R. W. S. KILADA. 1987. Interactive effect of diet and temperature on the growth of juvenile clams. J. Exp. Mar. Biol. Ecol. $113: 23-38$.
Legendre, L. Et P. Legendre. 1979. Écologie numérique. Tome 2 : La structure des données écologiques. Collection d'Ecologie, $\mathrm{n}^{\circ} 13$. Masson, Paris et les Presses de l'Université du Québec. 247 p.

ManN, R. ET S. J. GLOMB. 1978. The effect of temperature on growth and ammonia excretion of the Manila clam Tapes japonica. Estuar. Coastl. Mar. Sci. 6 : 335-339.

MANN, R. 1979. The effects of temperature on growth, physiology and gametogenesis in the Manila clam, Tapes philippinarum. J. Exp. Mar. Biol. Ecol. 38 : 121-134.

NELDER, J. A. ET R. MEAD. 1965. A simplex method for function minimization. Computer J. $7: 308-313$.

PaGe, H. M. ET D. M. HubBard. 1987. Temporal and spatial patterns of growth in mussels Mytilus edulis on an offshore platform: relationship to water temperature and food availability. J. Exp. Mar. Biol. Ecol. $111: 159-179$.

Parache, A. et H. Massé. 1987. Influence des facteurs du milieu sur le cycle biologique de Mytilus galloprovincialis en élevage sur corde, dans l'anse de Carteau (côte méditerranéenne française). Haliotis $16: 137-147$.

PETERSON, C. H. ET R. Black. 1987. Resource depletion by active suspension feeders on tidal flats: influence of local density and tidal elevation. Limnol. Oceanogr. $32: 143-166$.

Peyre, R., Y. Zanette et M. HÉral. 1980. Élevage de palourdes sous filet en milieu fermé. Science et Pêche, Bull. Inst. Pêches Marit., 307 : 1518.

Plante-Cuny, M. R. ET A. Bodoy. 1987. Relations trophiques entre microphytes benthiques ou planctoniques et mollusques bivalves endogés. Production et relations trophiques dans les écosystèmes marins. Deuxième colloque franco-soviétique Yalta, 1984. IFREMER, Act. Coll. 5 : 157 169.

SCHNUTE, J. 1982. A manual for easy non linear parameter estimation in fishery research with interactive microcomputer programs. Can. Techn. Rep. of Fish. and Aquat. Sci. $1140: 1-115$.

Sebens, K. P. 1982. The limits to indeterminate growth: an optimal size model applied to passive suspension-feeders. Ecology $63: 209-222$.

Sornin, J. M., M. Feullet, M. Héral, et J. M. Deslous-Paoli. 1983. Effets des biodépôts de l'huître Crassostrea gigas (Thunberg) sur l'accumulation de matières organiques dans les parcs du bassin de Marennes-Oléron. J. Moll. Stud. 12A : 185-197.

Sornin, J. M., M. Feuillet, M. Héral, et J. C. Fardeau. 1986. Influence des cultures d'huîtres Crassostrea gigas sur le cycle du phosphore en zone intertidale : rôle de la biodéposition. Oceanol. Acta. 9: 313-322.

Tomassone, R., E. LesQuY et C. Millier. 1983. La régression, nouveaux regards sur une ancienne méthode statistique. Éd. Masson, Paris. $180 \mathrm{p}$.

VAHL, O. 1980. Seasonal variations in seston and in the growth rate of the Iceland Scallop, Chlamys islandica from Bulsfjord $70^{\circ}$ N. J. Exp. Mar. Biol. Ecol. 48 : 195-204.

WARD, L. G., W. M. KEMP ET W. R. Boynton. 1984. The influence of waves and seagrass communities on suspended particulates in an estuarine embayment. Mar. Geol. 59: 85-103.

WARD, L. G. 1985. The influence of wind waves and tidal currents on sediment resuspension in Middle Chesapeake Bay. Geo. Marine Letters 5 : 71-75.

Wil.Dish, D. J. ET D. D. KRISTMANSON. 1979. Tidal energy and sublittoral macrobenthic animals in estuaries. J. Fish. Res. Board Can. $36: 1197-$ 1206. 